\title{
Dynamics of a stochastic service-resource mutualism model with Lévy noises and harvesting
}

\author{
Hui Wang ${ }^{\mathrm{a}}$, Chenxi Duª , Meng Liü, \\ a School of Mathematical Science, Huaiyin Normal University, Huaian 223300, P. R. China. \\ ${ }^{b}$ School of Mathematics and Statistics, Northeast Normal University, Jilin 130024, P. R. China.
}

Communicated by M. Bohner

\begin{abstract}
In this paper, we propose a stochastic service-resource mutualism model with Lévy noises and harvesting. Under some assumptions, we study several dynamical properties of the model. We first obtain the thresholds between persistence and extinction for both the service species and the resource species. Then we give sharp sufficient conditions for stability in distribution of the model. Finally, we establish sufficient and necessary criteria for the existence of the optimal harvesting policy. The optimal harvesting effort and maximum of sustainable yield are also obtained. Our results reveal that the persistence, extinction, stability in distribution and optimal harvesting strategy have close relationships with the random noises. (C)2017 All rights reserved.
\end{abstract}

Keywords: Service-resource mutualism model, white noise, Lévy jumps, persistence, optimal harvesting. 2010 MSC: 92D25, 60H10, 60H30.

\section{Introduction}

The investigation of the ecologically and economically reasonable harvesting strategies is one of the most significant issues in mathematical biology. Over-harvesting has driven many species to go to extinction. It is therefore important to establish the optimal harvesting strategy for a species or a community.

In recent years, the optimal harvesting problems have attracted much attention (see e.g., [8-10, 12, 14]). Under the famous catch-per unit effort (CPUE) hypothesis, Clark [10] gained the optimal harvesting strategy for a deterministic logistic population model with constant coefficients. Then the result in [10] was extended to the model with periodic coefficients by Fan and Wang [12]. Kar [14] established the optimal harvesting strategy for a prey-predator model with prey-refuge. Braverman and Mamdani [9] considered the optimal harvesting problems of single-species models with impulsive and continuous harvesting effects. Braverman and Braverman [8] analyzed the optimal harvesting problems of singlespecies models with diffusion.

In the real world, population models are inevitably affected by environmental perturbations (for example, cold wave, drought, fire). Consequently, it is interesting and important to consider the optimal harvesting problems of stochastic population models and to study the effects of environmental perturbations on the optimal harvesting strategy. Beddington and May [5] did pioneering work in this area. By

\footnotetext{
*Corresponding author

Email address: liumeng@hytc.edu.cn (Meng Liu)
} 
solving the corresponding Fokker-Planck equation, the authors [5] gained the optimal harvesting strategy for a stochastic Logistic equation. Zou et al. [29] proposed the ergodic method and studied the optimal harvesting problems of a stochastic Gompertz population model. Optimal harvesting problems of stochastic population models with time delay were investigated by $[16,19,20]$. Stochastic population models with harvesting and jumps were studied in [21, 26, 30].

On the other hand, service-resource mutualism is a usual phenomena in nature. A typical example is Zoochory [6]: plant provides resource (food) for animals, and animals provides service (dispersion of the seeds of the plants). Hence it is useful to study the optimal harvesting strategy of stochastic serviceresource mutualism models. However, as far as our knowledge is concerned, no results relative to this problem have been reported.

In this paper, we propose a stochastic service-resource mutualism model with harvesting in Section 2. Then we establish sharp sufficient conditions for the persistence in the mean and extinction for each species in Section 3, and study the stability in distribution of model in Section 4. Afterwards, we obtain the sufficient and necessary conditions for the existence of optimal harvesting policy, and gain the explicit forms of the optimal harvesting effect and the maximum of sustainable yield in Section 5. Finally, we introduce several simulation figures to illustrate the theoretical results, and give some concluding remarks in Section 6.

\section{The model}

Our model is based on the following Lotka-Volterra service-resource mutualism model $([24,25])$ :

$$
\left\{\begin{array}{l}
\frac{\mathrm{d} z_{1}(\mathrm{t})}{\mathrm{dt}}=z_{1}(\mathrm{t})\left[\mathrm{r}_{1}-\mathrm{m}_{11} z_{1}(\mathrm{t})+\mathrm{m}_{12} z_{2}(\mathrm{t})\right], \\
\frac{\mathrm{d} z_{2}(\mathrm{t})}{\mathrm{dt}}=z_{2}(\mathrm{t})\left[-\mathrm{r}_{2}+\mathrm{m}_{21} z_{1}(\mathrm{t})-\mathrm{m}_{22} z_{2}(\mathrm{t})\right],
\end{array}\right.
$$

where $z_{1}(t)$ stands for the population size of the resource species, and $z_{2}(t)$ represents the population size of the service species. $r_{1}>0$ is the growth rate of species $1, r_{2}>0$ is the death rate of species $2, m_{i i}>0$ is the intra-specific competition coefficient, and $m_{i j}>0(i \neq j)$ is the mutualistic rate, $i, j=1,2$.

According to the famous CPUE hypothesis ([10]), we assume that both the resource species and the service species are subject to harvesting with rates $h_{1} \geqslant 0$ and $h_{2} \geqslant 0$, respectively. Hence model (2.1) becomes:

$$
\left\{\begin{array}{l}
\frac{\mathrm{d} z_{1}(\mathrm{t})}{\mathrm{d} t}=z_{1}(\mathrm{t})\left[\mathrm{r}_{1}-\mathrm{m}_{11} z_{1}(\mathrm{t})+\mathrm{m}_{12} z_{2}(\mathrm{t})\right]-\mathrm{h}_{1} z_{1}(\mathrm{t}) \\
\frac{\mathrm{d} z_{2}(\mathrm{t})}{\mathrm{d} t}=z_{2}(\mathrm{t})\left[-\mathrm{r}_{2}+\mathrm{m}_{21} z_{1}(\mathrm{t})-\mathrm{m}_{22} z_{2}(\mathrm{t})\right]-\mathrm{h}_{2} z_{2}(\mathrm{t}) .
\end{array}\right.
$$

As said above, the growth of the species is often affected by various random noises. First of all, we consider the famous white noises. There are several approaches to introduce the white noises. In this paper we adopt the approach in $[5,7,13,17,18,27])$, that is to say, we assume that the white noises mainly affect the growth/death rates of the species, with

$$
r_{i}+\sigma_{i} \dot{B}_{i}(t)
$$

where $\dot{B}_{1}(t)$ and $\dot{B}_{2}(t)$ are white noises, namely, $\left\{B_{1}(t)\right\}_{t} \geqslant 0$ and $\left.\left\{B_{2}(t)\right)\right\}_{t} \geqslant 0$ are two standard Brownian motions defined on a complete probability space $\left(\Omega,\left\{\mathcal{F}_{t}\right\}_{t} \geqslant 0, P\right)$ with the filtration $\left\{\mathcal{F}_{t}\right\}, \sigma_{i}^{2}(i=1,2)$ denote the intensities of the white noises. Thereby, we obtain the following stochastic model:

$$
\left\{\begin{array}{l}
\mathrm{d} z_{1}(\mathrm{t})=z_{1}(\mathrm{t})\left[\mathrm{r}_{1}-\mathrm{h}_{1}-\mathrm{m}_{11} z_{1}(\mathrm{t})+\mathrm{m}_{12} z_{2}(\mathrm{t})\right] \mathrm{d} \mathrm{t}+\sigma_{1} z_{1}(\mathrm{t}) \mathrm{dB}_{1}(\mathrm{t}), \\
\mathrm{d} z_{2}(\mathrm{t})=z_{2}(\mathrm{t})\left[-\mathrm{r}_{2}-\mathrm{h}_{2}+\mathrm{m}_{21} z_{1}(\mathrm{t})-\mathrm{m}_{22} z_{2}(\mathrm{t})\right] \mathrm{d} \mathrm{t}+\sigma_{2} z_{2}(\mathrm{t}) \mathrm{dB} \mathrm{B}_{2}(\mathrm{t}) .
\end{array}\right.
$$

We are now in the position to incorporate another random noise, the Lévy noise, into account. As a matter of fact, the growth of the species in the natural world is often affected by many sudden random 
shocks (e.g., epidemics, earthquakes, toxic pollutants). For example, the Sandoz Chemical Accident, the Gulf of Mexico oil spill, and the SARS and the Fukushima Daiichi nuclear disaster. Generally speaking, these sudden random shocks can not be described by the white noises. Many researchers (see, e.g., $[2,18,21,26])$ have claimed that these sudden random shocks can be modeled by Lévy noises. Hence we gain the following model:

$$
\left\{\begin{array}{l}
\mathrm{d} z_{1}(\mathrm{t})=z_{1}\left(\mathrm{t}^{-}\right)\left[\mathrm{r}_{1}-\mathrm{h}_{1}-\mathrm{m}_{11} z_{1}\left(\mathrm{t}^{-}\right)+\mathrm{m}_{12} z_{2}\left(\mathrm{t}^{-}\right)\right] \mathrm{dt}+\sigma_{1} z_{1}\left(\mathrm{t}^{-}\right) \mathrm{dB} B_{1}(\mathrm{t})+z_{1}\left(\mathrm{t}^{-}\right) \int_{\mathbb{X}} \xi_{1}(\mathrm{x}) \tilde{\mathrm{Q}}(\mathrm{dt}, \mathrm{dx}), \\
\mathrm{d} z_{2}(\mathrm{t})=z_{2}\left(\mathrm{t}^{-}\right)\left[-\mathrm{r}_{2}-\mathrm{h}_{2}+\mathrm{m}_{21} z_{1}\left(\mathrm{t}^{-}\right)-\mathrm{m}_{22} z_{2}\left(\mathrm{t}^{-}\right)\right] \mathrm{dt}+\sigma_{2} z_{2}\left(\mathrm{t}^{-}\right) \mathrm{dB}_{2}(\mathrm{t})+z_{2}\left(\mathrm{t}^{-}\right) \int_{\mathbb{X}} \xi_{2}(\mathrm{x}) \tilde{\mathrm{Q}}(\mathrm{dt}, \mathrm{dx})
\end{array}\right.
$$

where $z_{\mathfrak{i}}\left(t^{-}\right)$is the left limit of $z_{\mathfrak{i}}(t), \tilde{Q}(d t, d x)=Q(d t, d x)-\beta(d x) d t, Q$ is a Poisson counting measure, $\mathbb{X}$ is a measurable subset of $(0,+\infty)$, and $\beta$ is the characteristic measure of $Q$ with $\beta(\mathbb{X})<+\infty$. Throughout this paper, we always suppose that $Q, B_{1}(t)$, and $B_{2}(t)$ are independent, and $1+\xi_{i}(x)>0, x \in \mathbb{X}, i=1,2$.

\section{Persistence and extinction}

First of all, we define some notations and introduce an assumption.

$$
R_{+}^{2}=\left\{x \in R^{2} \mid x_{i}>0, i=1,2\right\}, \eta_{i}=\frac{\sigma_{i}^{2}}{2}+\int_{\mathbb{X}}\left(\xi_{i}(x)-\ln \left(1+\xi_{i}(x)\right)\right) \beta(d x), i=1,2,
$$

$b_{1}=r_{1}-\eta_{1}-h_{1}, b_{2}=r_{2}+\eta_{2}+h_{2}, M=m_{11} m_{22}-m_{12} m_{21}, M_{1}=b_{1} m_{22}-b_{2} m_{12}, M_{2}=b_{1} m_{21}-b_{2} m_{11}$.

Assumption 3.1. There is a constant $\tilde{\mathrm{c}}>0$ such that

$$
\int_{\mathbb{X}}\left[\ln \left(1+\xi_{i}(x)\right)\right]^{2} \beta(d x)<\tilde{c}, \int_{\mathbb{X}} \xi_{i}^{2}(x) \beta(d x)<\tilde{c}, i=1,2,
$$

which means that the intensities of the Lévy noises are not too large.

Lemma 3.2. If $M>0$, then for any initial value $z(0) \in R_{+}^{2}$, model (2.2) has a unique global positive solution $z(t)=\left(z_{1}(t), z_{2}(t)\right)^{\mathrm{T}}$ almost surely (a.s.). If moreover, Assumption 3.1 holds, then

$$
\lim _{\mathrm{t} \rightarrow+\infty} \frac{\ln z_{\mathfrak{i}}(\mathrm{t})}{\ln \mathrm{t}} \leqslant 1, \text { a.s., } \mathrm{i}=1,2,
$$

and for any $\mathrm{p}>0$, there exists a constant $\mathrm{c}=\mathrm{c}(\mathrm{p})>0$ such that

$$
\limsup _{t \rightarrow+\infty} \mathbb{E}\left[z_{i}^{p}(t)\right] \leqslant c, \quad i=1,2 .
$$

Proof. The proofs of the existence and uniqueness of the global positive solution and (3.1) are similar to these in [22] (Lemmas 3 and 4), and hence are omitted.

The proof of (3.2) is similar to that in [2] (Theorem 3.1) and we omit it too.

Lemma 3.3 ([22]). Suppose that $\mathrm{y}(\mathrm{t}):[0,+\infty) \rightarrow \mathrm{R}_{+}$is a stochastic process, and let Assumption 3.1 hold.

(a) If there exist two positive constants $\mathrm{T}$ and $\delta_{0}$ such that

$$
\ln y(t) \leqslant \delta t-\delta_{0} \int_{0}^{t} y(s) d s+\sum_{i=1}^{2} \sigma_{i} B_{i}(t)+\sum_{i=1}^{2} \delta_{i} \int_{0}^{t} \int_{\mathbb{X}} \ln \left(1+\xi_{i}(x)\right) \tilde{Q}(\mathrm{~d} s, d x) \text { a.s. }
$$

for all $\mathrm{t} \geqslant \mathrm{T}$, where $\sigma_{i}$ and $\delta_{i}$ are constants, then

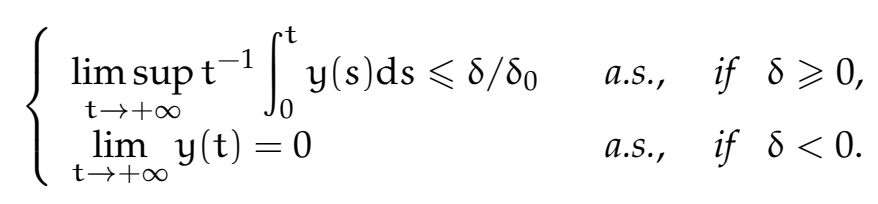


(b) If there exist three positive constants $T, \delta$, and $\delta_{0}$ such that

$$
\ln y(t) \geqslant \delta t-\delta_{0} \int_{0}^{t} y(s) d s+\sum_{i=1}^{2} \sigma_{i} B_{i}(t)+\sum_{i=1}^{2} \delta_{i} \int_{0}^{t} \int_{\mathbb{X}} \ln \left(1+\xi_{i}(x)\right) \tilde{Q}(d s, d x) \text { a.s. }
$$

for all $\mathrm{t} \geqslant \mathrm{T}$, then

$$
\liminf _{t \rightarrow+\infty} t^{-1} \int_{0}^{t} y(s) d s \geqslant \delta / \delta_{0} \quad \text { a.s.. }
$$

We are now in the position to give our first main result.

Theorem 3.4. For model (2.2), if $\mathrm{M}>0$ and Assumption 3.1 holds, then

(I) if $\mathrm{M}_{2}>0$, then both $z_{1}$ and $z_{2}$ are persistent in the mean, i.e.,

$$
\lim _{\mathrm{t} \rightarrow+\infty} \mathrm{t}^{-1} \int_{0}^{\mathrm{t}} z_{1}(\mathrm{~s}) \mathrm{d} s=\frac{\mathrm{M}_{1}}{\mathrm{M}}, \lim _{\mathrm{t} \rightarrow+\infty} \mathrm{t}^{-1} \int_{0}^{\mathrm{t}} z_{2}(\mathrm{~s}) \mathrm{d} s=\frac{\mathrm{M}_{2}}{\mathrm{M}}, \text { a.s.; }
$$

(II) if $\mathrm{M}_{2}=0$, then the service species is non-persistent in the mean a.s.: $\lim _{\mathrm{t} \rightarrow+\infty} \mathrm{t}^{-1} \int_{0}^{\mathrm{t}} z_{2}(\mathrm{~s}) \mathrm{d} \mathrm{s}=0$ a.s., and

$$
\lim _{\mathrm{t} \rightarrow+\infty} \mathrm{t}^{-1} \int_{0}^{\mathrm{t}} z_{1}(\mathrm{~s}) \mathrm{d} s=\mathrm{b}_{1} / \mathrm{m}_{11} \text {, a.s. }
$$

(III) if $\mathrm{M}_{2}<0$,

(i) if $\mathrm{b}_{1}>0$, then (3.4) holds and the service species dies out a.s., i.e., $\lim _{\mathrm{t} \rightarrow+\infty} z_{2}(\mathrm{t})=0$, a.s.;

(ii) if $\mathrm{b}_{1}=0$, then the service species dies out a.s., and the resource species is non-persistent in the mean a.s.: $\lim _{\mathrm{t} \rightarrow+\infty} \mathrm{t}^{-1} \int_{0}^{\mathrm{t}} z_{1}(\mathrm{~s}) \mathrm{d} \mathrm{s}=0$ a.s.;

(iii) if $\mathrm{b}_{1}<0$, then both the resource species and the service species die out a.s..

Proof. According to Itô's formula (see, e.g., [15]), one can see that

$$
\begin{aligned}
& \mathrm{d} z_{1}(\mathrm{t})=\left[\mathrm{b}_{1}-\mathrm{m}_{11} z_{1}(\mathrm{t})+\mathrm{m}_{12} z_{2}(\mathrm{t})\right] \mathrm{d} \mathrm{t}+\sigma_{1} \mathrm{~dB}_{1}(\mathrm{t})+\int_{\mathbb{X}} \ln \left(1+\xi_{1}(\mathrm{x})\right) \tilde{\mathrm{Q}}(\mathrm{dt}, \mathrm{d} \mathrm{x}), \\
& \mathrm{d} z_{2}(\mathrm{t})=\left[-\mathrm{b}_{2}+\mathrm{m}_{21} z_{1}(\mathrm{t})-\mathrm{m}_{22} z_{2}(\mathrm{t})\right] \mathrm{d} \mathrm{t}+\sigma_{2} \mathrm{~dB}_{2}(\mathrm{t})+\int_{\mathbb{X}} \ln \left(1+\xi_{2}(\mathrm{x})\right) \tilde{\mathrm{Q}}(\mathrm{d} \mathrm{t}, \mathrm{d} x) .
\end{aligned}
$$

Consequently,

$$
\begin{aligned}
\mathrm{t}^{-1} \ln \frac{z_{1}(\mathrm{t})}{z_{1}(0)}= & \mathrm{b}_{1}-\mathrm{m}_{11} \mathrm{t}^{-1} \int_{0}^{\mathrm{t}} z_{1}(\mathrm{~s}) \mathrm{d} s+\mathrm{m}_{12} \mathrm{t}^{-1} \int_{0}^{\mathrm{t}} z_{2}(\mathrm{~s}) \mathrm{d} \mathrm{s} \\
& +\sigma_{1} \mathrm{t}^{-1} \mathrm{~B}_{1}(\mathrm{t})+\mathrm{t}^{-1} \int_{0}^{\mathrm{t}} \int_{\mathbb{X}} \ln \left(1+\xi_{1}(\mathrm{x})\right) \tilde{\mathrm{Q}}(\mathrm{d} s, \mathrm{~d} \mathrm{x}), \\
\mathrm{t}^{-1} \ln \frac{z_{2}(\mathrm{t})}{z_{2}(0)}= & -\mathrm{b}_{2}+\mathrm{m}_{21} \mathrm{t}^{-1} \int_{0}^{\mathrm{t}} z_{1}(\mathrm{~s}) \mathrm{d} s-\mathrm{m}_{22} \mathrm{t}^{-1} \int_{0}^{\mathrm{t}} z_{2}(\mathrm{~s}) \mathrm{d} \mathrm{s} \\
& +\sigma_{2} \mathrm{t}^{-1} \mathrm{~B}_{2}(\mathrm{t})+\mathrm{t}^{-1} \int_{0}^{\mathrm{t}} \int_{\mathbb{X}} \ln \left(1+\xi_{2}(\mathrm{x})\right) \tilde{\mathrm{Q}}(\mathrm{ds}, \mathrm{d} \mathrm{x}) .
\end{aligned}
$$

(I). By $(3.5) \times \mathrm{m}_{21}+(3.6) \times \mathrm{m}_{11}$, we have

$$
\begin{aligned}
\mathrm{t}^{-1} \mathrm{~m}_{21} & \ln \frac{z_{1}(\mathrm{t})}{z_{1}(0)}+\mathrm{t}^{-1} \mathrm{~m}_{11} \ln \frac{z_{2}(\mathrm{t})}{z_{2}(0)} \\
= & \mathrm{b}_{1} \mathrm{~m}_{21}-\mathrm{b}_{2} \mathrm{~m}_{11}-M \mathrm{t}^{-1} \int_{0}^{\mathrm{t}} z_{2}(\mathrm{~s}) \mathrm{d} \mathrm{s}+\sigma_{1} \mathrm{~m}_{21} \mathrm{t}^{-1} \mathrm{~B}_{1}(\mathrm{t})+\sigma_{2} \mathrm{~m}_{11} \mathrm{t}^{-1} \mathrm{~B}_{2}(\mathrm{t}) \\
& +\mathrm{m}_{21} \mathrm{t}^{-1} \int_{0}^{\mathrm{t}} \int_{\mathbb{X}} \ln \left(1+\xi_{1}(\mathrm{x})\right) \tilde{Q}(\mathrm{ds}, \mathrm{d} \mathrm{x})+\mathrm{m}_{11} \mathrm{t}^{-1} \int_{0}^{\mathrm{t}} \int_{\mathbb{X}} \ln \left(1+\xi_{2}(\mathrm{x})\right) \tilde{\mathrm{Q}}(\mathrm{d} \mathrm{s}, \mathrm{d} \mathrm{x}) .
\end{aligned}
$$

In view of (3.1), for arbitrary $\varepsilon \in\left(0, \min \left\{1, M_{2}\right\}\right)$, there exists a measurable set $\Omega_{\varepsilon} \subset \Omega$ with $\mathrm{P}\left(\Omega_{\varepsilon}\right) \geqslant 1-\varepsilon$ 
and $\mathrm{a} T=T(\varepsilon)>0$ such that for $\omega \in \Omega_{\varepsilon}$ and $t \geqslant T$,

$$
\mathrm{t}^{-1} \mathrm{~m}_{21} \ln \frac{z_{1}(\mathrm{t})}{z_{1}(0)}-\mathrm{t}^{-1} \mathrm{~m}_{11} \ln z_{2}(0) \leqslant \varepsilon .
$$

When this inequality is used in (3.7), one can see that for $t \geqslant T$,

$$
\begin{aligned}
\mathrm{t}^{-1} \mathrm{~m}_{11} \ln z_{2}(\mathrm{t}) \geqslant & \mathrm{M}_{2}-\varepsilon-M \mathrm{t}^{-1} \int_{0}^{\mathrm{t}} z_{2}(\mathrm{~s}) \mathrm{d} \mathrm{s}+\sigma_{1} \mathrm{~m}_{21} \mathrm{t}^{-1} \mathrm{~B}_{1}(\mathrm{t})+\sigma_{2} \mathrm{~m}_{11} \mathrm{t}^{-1} \mathrm{~B}_{2}(\mathrm{t}) \\
& +\mathrm{m}_{21} \mathrm{t}^{-1} \int_{0}^{\mathrm{t}} \int_{\mathbb{X}} \ln \left(1+\xi_{1}(\mathrm{x})\right) \tilde{\mathrm{Q}}(\mathrm{d} s, \mathrm{~d} \mathrm{x})+\mathrm{m}_{11} \mathrm{t}^{-1} \int_{0}^{\mathrm{t}} \int_{\mathbb{X}} \ln \left(1+\xi_{2}(\mathrm{x})\right) \tilde{\mathrm{Q}}(\mathrm{d} \mathrm{s}, \mathrm{d} \mathrm{x}) .
\end{aligned}
$$

It then follows from (b) in Lemma 3.3 and the arbitrariness of $\varepsilon$ that

$$
\liminf _{t \rightarrow+\infty} t^{-1} \int_{0}^{t} z_{2}(s) d s \geqslant M_{2} / M, \text { a.s.. }
$$

In the same way, one can prove that

$$
\liminf _{t \rightarrow+\infty} t^{-1} \int_{0}^{t} z_{1}(s) d s \geqslant M_{1} / M, \text { a.s.. }
$$

To prove the desired assertion (3.3), we need only to show that

$$
\limsup _{t \rightarrow+\infty} t^{-1} \int_{0}^{t} z_{i}(s) d s \leqslant M_{i} / M, \text { a.s., } i=1,2 .
$$

In fact, according to (3.5), we can observe that for sufficiently large $t$,

$$
\begin{aligned}
\mathrm{t}^{-1} \ln z_{1}(\mathrm{t}) \leqslant & \mathrm{b}_{1}+\varepsilon-\mathrm{m}_{11} \mathrm{t}^{-1} \int_{0}^{\mathrm{t}} z_{1}(\mathrm{~s}) \mathrm{d} \mathrm{s}+\mathrm{m}_{12} \limsup _{\mathrm{t} \rightarrow+\infty}\left[\mathrm{t}^{-1} \int_{0}^{\mathrm{t}} z_{2}(\mathrm{~s}) \mathrm{d} \mathrm{s}\right] \\
& +\sigma_{1} \mathrm{t}^{-1} \mathrm{~B}_{1}(\mathrm{t})+\mathrm{t}^{-1} \int_{0}^{\mathrm{t}} \int_{\mathbb{X}} \ln \left(1+\xi_{1}(\mathrm{x})\right) \tilde{\mathrm{Q}}(\mathrm{d} s, \mathrm{~d} x) .
\end{aligned}
$$

In view of (3.8), the resource species does not go to extinction. An application of Lemma 3.3 gives

$$
\mathrm{b}_{1}+\varepsilon+\mathrm{m}_{12} \limsup _{\mathrm{t} \rightarrow+\infty}\left[\mathrm{t}^{-1} \int_{0}^{\mathrm{t}} z_{2}(\mathrm{~s}) \mathrm{d} \mathrm{s}\right]>0 .
$$

It then follows from Lemma 3.3 and the arbitrariness of $\varepsilon$ that

$$
\limsup _{t \rightarrow+\infty}\left[t^{-1} \int_{0}^{t} z_{1}(s) d s\right] \leqslant \frac{b_{1}+m_{12} \limsup _{t \rightarrow+\infty}\left[t^{-1} \int_{0}^{t} z_{2}(s) d s\right]}{m_{11}}, \text { a.s.. }
$$

Consequently,

$$
m_{11} \limsup _{t \rightarrow+\infty}\left[t^{-1} \int_{0}^{t} z_{1}(s) d s\right]-m_{12} \limsup _{t \rightarrow+\infty}\left[t^{-1} \int_{0}^{t} z_{2}(s) d s\right] \leqslant b_{1}, \text { a.s.. }
$$

Similarly, by (3.6), we gain

$$
\mathrm{m}_{22} \limsup _{\mathrm{t} \rightarrow+\infty}\left[\mathrm{t}^{-1} \int_{0}^{\mathrm{t}} z_{2}(\mathrm{~s}) \mathrm{ds}\right]-\mathrm{m}_{21} \limsup _{\mathrm{t} \rightarrow+\infty}\left[\mathrm{t}^{-1} \int_{0}^{\mathrm{t}} z_{1}(\mathrm{~s}) \mathrm{ds}\right] \leqslant-\mathrm{b}_{2} \text {, a.s.. }
$$

Solving (3.10) and (3.11) leads to the required assertion (3.9). 
(II). Note that $M_{2}=b_{1} m_{21}-b_{2} m_{11}=0$, hence $b_{1}>0$. According to (3.5), for arbitrary $\varepsilon \in\left(0, \min \left\{1, b_{1}\right\}\right)$, there exists a measurable set $\Omega_{\varepsilon} \subset \Omega$ with $\mathrm{P}\left(\Omega_{\varepsilon}\right) \geqslant 1-\varepsilon$ and a $\mathrm{T}=\mathrm{T}(\varepsilon)>0$ such that for $\omega \in \Omega_{\varepsilon}$ and $t \geqslant T$,

$$
\mathrm{t}^{-1} \ln z_{1}(\mathrm{t}) \geqslant \mathrm{b}_{1}-\varepsilon-\mathrm{m}_{11} \mathrm{t}^{-1} \int_{0}^{\mathrm{t}} z_{1}(\mathrm{~s}) \mathrm{d} \mathrm{s}+\sigma_{1} \mathrm{t}^{-1} \mathrm{~B}_{1}(\mathrm{t})+\mathrm{t}^{-1} \int_{0}^{\mathrm{t}} \int_{\mathbb{X}} \ln \left(1+\xi_{1}(\mathrm{x})\right) \tilde{\mathrm{Q}}(\mathrm{d} s, \mathrm{~d} \mathrm{x}) .
$$

It then follows from Lemma 3.3 and the arbitrariness of $\varepsilon$ that

$$
\liminf _{t \rightarrow+\infty} t^{-1} \int_{0}^{t} z_{1}(s) d s \geqslant b_{1} / m_{11} \text {, a.s.. }
$$

Hence the resource species does not go to extinction, and then we get (3.10). Now we are in the position to show

$$
\limsup _{t \rightarrow+\infty} t^{-1} \int_{0}^{t} z_{2}(s) \mathrm{d} s=0 \text {, a.s.. }
$$

Denote by

$$
\mathrm{D}_{1}=\left\{\limsup _{\mathrm{t} \rightarrow+\infty} \mathrm{t}^{-1} \int_{0}^{\mathrm{t}} z_{2}(\mathrm{~s}) \mathrm{d} \mathrm{s}>0\right\} .
$$

Assume that $\mathrm{P}\left(\mathrm{D}_{1}\right)>0$. For any $\omega \in \mathrm{D}_{1}$, (3.11) holds. Substituting (3.10) into (3.11) leads to

$$
\begin{aligned}
\mathrm{m}_{22}\left[\limsup _{t \rightarrow+\infty} t^{-1} \int_{0}^{t} z_{2}(s, \omega) d s\right] & \leqslant-b_{2}+m_{21}\left[\limsup _{t \rightarrow+\infty} t^{-1} \int_{0}^{t} z_{1}(s, \omega) d s\right] \\
& \leqslant-b_{2}+\frac{b_{1}+m_{12}\left[\limsup _{t \rightarrow+\infty} t^{-1} \int_{0}^{t} z_{2}(s, \omega) d s\right]}{m_{11}} m_{21} .
\end{aligned}
$$

Consequently,

$$
\limsup _{t \rightarrow+\infty} t^{-1} \int_{0}^{t} z_{2}(s, \omega) d s \leqslant M_{2} / M .
$$

Since $M_{2}=0$, then the contradiction arises. Thereby one gets (3.12). Substituting (3.12) into (3.10) results in

$$
\limsup _{t \rightarrow+\infty} t^{-1} \int_{0}^{t} z_{1}(s) d s \leqslant b_{1} / m_{11} \text {, a.s.. }
$$

Then (3.4) follows.

(III). (i) Note that $b_{1}>0$, it then follows from the proof of (II) that (3.13) holds. And hence (3.4) holds. Substituting (3.4) into (3.6), we can observe that for sufficiently large $t$,

$$
\mathrm{t}^{-1} \ln z_{2}(\mathrm{t}) \leqslant \frac{\mathrm{M}_{2}}{\mathrm{~m}_{11}}+\varepsilon-\mathrm{m}_{22} \mathrm{t}^{-1} \int_{0}^{\mathrm{t}} z_{2}(\mathrm{~s}) \mathrm{d} \mathrm{s}+\sigma_{2} \mathrm{t}^{-1} \mathrm{~B}_{2}(\mathrm{t})+\mathrm{t}^{-1} \int_{0}^{\mathrm{t}} \int_{\mathbb{X}} \ln \left(1+\xi_{2}(\mathrm{x})\right) \tilde{\mathrm{Q}}(\mathrm{ds}, \mathrm{d} \mathrm{x}) .
$$

where $M_{2} / m_{11}+\varepsilon<0$. It then follows from Lemma 3.3 and the arbitrariness of $\varepsilon$ that $\lim _{t \rightarrow+\infty} z_{2}(t)=0$, a.s..

Finally, let us show (ii) and (iii) together. Firstly, let us prove that $\lim _{t \rightarrow+\infty} z_{2}(t)=0$ a.s.. Let

$$
D_{2}=\left\{\limsup _{t \rightarrow+\infty} t^{-1} \int_{0}^{t} z_{1}(s) d s>0\right\}, D_{3}=\left\{\limsup _{t \rightarrow+\infty} t^{-1} \int_{0}^{t} z_{1}(s) d s=0\right\} .
$$

Hence, $P\left(D_{2}\right)+P\left(D_{3}\right)=1$. For arbitrary $\omega \in D_{2}$, in view of (II), we can see that

$$
\lim _{t \rightarrow+\infty} z_{2}(t, \omega)=0 \text {. }
$$


For every $\omega \in S_{3}$, by (3.6), we have

$$
\mathrm{t}^{-1} \ln z_{2}(\mathrm{t}, \omega) \leqslant-\mathrm{b}_{2}+\varepsilon-\mathrm{m}_{22} \mathrm{t}^{-1} \int_{0}^{\mathrm{t}} z_{2}(\mathrm{~s}) \mathrm{ds}+\mathrm{\sigma}_{2} \mathrm{t}^{-1} \mathrm{~B}_{2}(\mathrm{t})+\mathrm{t}^{-1} \int_{0}^{\mathrm{t}} \int_{\mathbb{X}} \ln \left(1+\xi_{2}(\mathrm{x})\right) \tilde{\mathrm{Q}}(\mathrm{ds}, \mathrm{dx}),
$$

where $\varepsilon<b_{2}$. An application of Lemma 3.3 and the arbitrariness of $\varepsilon$ gives

$$
\lim _{t \rightarrow+\infty} z_{2}(t, \omega)=0
$$

Thereby, $\lim _{\mathrm{t} \rightarrow+\infty} z_{2}(\mathrm{t})=0$, a.s..

Substituting $\lim _{t \rightarrow+\infty} z_{2}(t)=0$ into (3.5) yields

$$
\mathrm{t}^{-1} \ln z_{1}(\mathrm{t}) \leqslant \mathrm{b}_{1}+\varepsilon-\mathrm{m}_{11} \mathrm{t}^{-1} \int_{0}^{\mathrm{t}} z_{1}(\mathrm{~s}) \mathrm{d} \mathrm{s}+\sigma_{1} \mathrm{t}^{-1} \mathrm{~B}_{1}(\mathrm{t})+\mathrm{t}^{-1} \int_{0}^{\mathrm{t}} \int_{\mathbb{X}} \ln \left(1+\xi_{1}(\mathrm{x})\right) \tilde{\mathrm{Q}}(\mathrm{d} s, \mathrm{~d} \mathrm{x}) .
$$

If $b_{1}<0$, then by Lemma 3.3 and the arbitrariness of $\varepsilon$,

$$
\lim _{\mathrm{t} \rightarrow+\infty} z_{1}(\mathrm{t})=0 \text {, a.s.. }
$$

If $b_{1}=0$, then according to Lemma 3.3 and the arbitrariness of $\varepsilon$,

$$
\limsup _{t \rightarrow+\infty} t^{-1} \int_{0}^{t} z_{1}(s) d s \leqslant b_{1}=0 \text {, a.s.. }
$$

This completes the proof.

Remark 3.5. According to Theorem 3.4, we can see that both the service species and the resource species are persistent if and only if $M_{2}>0$.

\section{Stability in distribution}

Definition 4.1. If there exists a unique probability measure $\chi(\cdot)$ with support $R_{+}^{2}$ such that for arbitrary $z(0) \in R_{+}^{2}$, the transition probability $p(t, z(0), \cdot)$ of $z(t)$ converges weakly to $\chi(\cdot)$ with $t \rightarrow+\infty$, then model (2.2) is said to be stable in distribution (SID).

Assumption 4.2. $m_{11}>m_{21}, m_{22}>m_{12}$. This assumption is a technical assumption to make the proof work.

Theorem 4.3. Let Assumptions 3.1 and 4.2 hold. If $\mathrm{M}_{2}>0$, then model (2.2) is SID.

Proof. Let $z(t ; z(0))$ and $z(t ; \tilde{z}(0))$ stand for two solutions of $(2.2)$ with $z(0) \in R_{+}^{2}$ and $\tilde{z}(0) \in R_{+}^{2}$, respectively. Define

$$
\mathrm{U}(\mathrm{t})=\sum_{\mathfrak{i}=1}^{2}\left|\ln z_{\mathfrak{i}}(\mathrm{t} ; z(0))-\ln z_{\mathfrak{i}}(\mathrm{t} ; \tilde{z}(0))\right| .
$$

It then follows from Itô's formula (see e.g., [15]) that

$$
\begin{aligned}
\mathrm{du}(\mathrm{t})= & \operatorname{sgn}\left(z_{1}(\mathrm{t} ; z(0))-z_{1}(\mathrm{t} ; \tilde{z}(0))\right)\left[-\mathrm{m}_{11}\left(z_{1}(\mathrm{t} ; z(0))-z_{1}(\mathrm{t} ; \tilde{z}(0))\right)+\mathrm{m}_{12}\left(z_{2}(\mathrm{t} ; z(0))-z_{2}(\mathrm{t} ; \tilde{z}(0))\right)\right] \mathrm{dt} \\
& +\operatorname{sgn}\left(z_{2}(\mathrm{t} ; z(0))-z_{2}(\mathrm{t} ; \tilde{z}(0))\right)\left[\mathrm{m}_{21}\left(z_{1}(\mathrm{t} ; z(0))-z_{1}(\mathrm{t} ; \tilde{z}(0))\right)-\mathrm{m}_{22}\left(z_{2}(\mathrm{t} ; z(0))-z_{2}(\mathrm{t} ; \tilde{z}(0))\right)\right] \mathrm{dt} \\
\leqslant & -\left(\mathrm{m}_{11}-\mathrm{m}_{21}\right)\left|z_{1}(\mathrm{t} ; z(0))-z_{1}(\mathrm{t} ; \tilde{z}(0))\right| \mathrm{dt}-\left(\mathrm{m}_{22}-\mathrm{m}_{12}\right)\left|z_{2}(\mathrm{t} ; z(0))-z_{2}(\mathrm{t} ; \tilde{z}(0))\right| \mathrm{dt} .
\end{aligned}
$$


That is to say,

$$
\begin{aligned}
0 \leqslant \mathbb{E}(\mathrm{U}(\mathrm{t})) \leqslant & \mathrm{u}(0)-\left(\mathrm{m}_{11}-\mathrm{m}_{21}\right) \int_{0}^{\mathrm{t}} \mathbb{E}\left|z_{1}(\mathrm{~s} ; z(0))-z_{1}(\mathrm{~s} ; \tilde{z}(0))\right| \mathrm{d} s \\
& -\left(\mathrm{m}_{22}-\mathrm{m}_{12}\right) \int_{0}^{\mathrm{t}} \mathbb{E}\left|z_{2}(s ; z(0))-z_{2}(\mathrm{~s} ; \tilde{z}(0))\right| \mathrm{d} s .
\end{aligned}
$$

Since $m_{11}>m_{21}, m_{22}>m_{12}$ and $u(0)<+\infty$, then we have

$$
\left(\mathrm{m}_{11}-\mathrm{m}_{21}\right) \int_{0}^{\mathrm{t}} \mathbb{E}\left|z_{1}(s ; z(0))-z_{1}(s ; \tilde{z}(0))\right| \mathrm{d} s+\left(\mathrm{m}_{22}-\mathrm{m}_{12}\right) \int_{0}^{\mathrm{t}} \mathbb{E}\left|z_{2}(s ; z(0))-z_{2}(s ; \tilde{z}(0))\right| \mathrm{d} s \leqslant \mathrm{U}(0)<+\infty \text {. }
$$

Thereby,

According to (2.2),

$$
\mathbb{E}\left|z_{\mathfrak{i}}(\mathrm{t} ; z(0))-z_{\mathfrak{i}}(\mathrm{t} ; \tilde{z}(0))\right| \in \mathrm{L}^{1}[0, \infty), \mathfrak{i}=1,2
$$

$$
\begin{aligned}
& \mathbb{E}\left(z_{1}(\mathrm{t})\right)=z_{1}(0)+\int_{0}^{\mathrm{t}}\left[\left(\mathrm{r}_{1}-\mathrm{h}_{1}\right) \mathbb{E}\left(z_{1}(\mathrm{~s})\right)-\mathrm{m}_{11} \mathbb{E}\left(z_{1}^{2}(\mathrm{~s})\right)+\mathrm{m}_{12} \mathbb{E}\left(z_{1}(\mathrm{~s}) z_{2}(\mathrm{~s})\right)\right] \mathrm{d} s, \\
& \mathbb{E}\left(z_{2}(\mathrm{t})\right)=z_{2}(0)+\int_{0}^{\mathrm{t}}\left[\left(-\mathrm{r}_{2}-\mathrm{h}_{2}\right) \mathbb{E}\left(z_{2}(\mathrm{~s})\right)-\mathrm{m}_{22} \mathbb{E}\left(z_{2}^{2}(\mathrm{~s})\right)+\mathrm{m}_{21} \mathbb{E}\left(z_{1}(\mathrm{~s}) z_{2}(\mathrm{~s})\right)\right] \mathrm{d} \mathrm{s} .
\end{aligned}
$$

Hence $\mathbb{E}\left(z_{1}(t)\right)$ and $\mathbb{E}\left(z_{2}(t)\right)$ are continuously differentiable functions. In view of (3.2),

$$
\begin{aligned}
\frac{d \mathbb{E}\left(z_{1}(t)\right)}{\mathrm{dt}} & =\left(\mathrm{r}_{1}-\mathrm{h}_{1}\right) \mathbb{E}\left(z_{1}(\mathrm{t})\right)-\mathrm{m}_{11} \mathbb{E}\left(z_{1}^{2}(\mathrm{t})\right)+\mathrm{m}_{12} \mathbb{E}\left(z_{1}(\mathrm{t}) z_{2}(\mathrm{t})\right) \\
& \leqslant \mathrm{r}_{1} \mathbb{E}\left(z_{1}(\mathrm{t})\right)+\frac{\mathrm{m}_{12}}{2} \mathbb{E}\left(z_{1}^{2}(\mathrm{t})+z_{2}^{2}(\mathrm{t})\right) \leqslant \mathrm{c}_{1}, \\
\frac{\mathrm{d} \mathbb{E}\left(z_{2}(\mathrm{t})\right)}{\mathrm{dt}} & =\left(-r_{2}-\mathrm{h}_{2}\right) \mathbb{E}\left(z_{2}(\mathrm{t})\right)-\mathrm{m}_{22} \mathbb{E}\left(z_{2}^{2}(\mathrm{t})\right)+\mathrm{m}_{21} \mathbb{E}\left(z_{1}(\mathrm{t}) z_{2}(\mathrm{t})\right) \leqslant \frac{\mathrm{m}_{21}}{2} \mathbb{E}\left(z_{1}^{2}(\mathrm{t})+z_{2}^{2}(\mathrm{t})\right) \leqslant \mathrm{c}_{1},
\end{aligned}
$$

where $c_{1}$ is a positive constant. Consequently, $\mathbb{E}\left(z_{1}(t)\right)$ and $\mathbb{E}\left(z_{2}(t)\right)$ are uniformly continuous functions. An application of Barbalat's result [3] yields

$$
\lim _{\mathrm{t} \rightarrow+\infty} \mathbb{E}\left|z_{\mathfrak{i}}(\mathrm{t} ; z(0))-z_{\mathfrak{i}}(\mathrm{t} ; \tilde{z}(0))\right|=0, \mathfrak{i}=1,2
$$

Denote by $P(t, z(0), \Upsilon)$ the probability of $\{z(t ; z(0)) \in \Upsilon\}$ with initial value $z(0) \in R_{+}^{2}$, where $\Upsilon$ is a Borel set. According to (3.2) and Chebyshev's inequality (see e.g., [23]), it is easy to see that $\{p(t, z(0), \cdot)\}$ is tight ([23]).

Denote by $\mathscr{P}\left(\mathrm{R}_{+}^{2}\right)$ all the probability measures on $\mathrm{R}_{+}^{2}$. For arbitrary $\mathrm{P}_{1}, \mathrm{P}_{2} \in \mathscr{P}$, define the Kantorovich metric as follows:

$$
\mathrm{d}_{\Phi}\left(\mathrm{P}_{1}, \mathrm{P}_{2}\right)=\sup _{\phi \in \Phi}\left|\int_{R_{+}^{2}} \phi(\theta) \mathrm{P}_{1}(\mathrm{~d} \theta)-\int_{R_{+}^{2}} \phi(\theta) \mathrm{P}_{2}(\mathrm{~d} \theta)\right|
$$

where

$$
\Phi=\left\{\phi: R_{+}^{2} \rightarrow R|| \phi\left(\theta_{1}\right)-\phi\left(\theta_{2}\right)\left|\leqslant\left\|\theta_{1}-\theta_{2}\right\|,\right| \phi(\cdot) \mid \leqslant 1\right\} .
$$

For all $\phi \in \Phi$ and $t, s>0$, we compute that

$$
\begin{aligned}
|\mathbb{E} \phi(z(t+s ; z(0)))-\mathbb{E} \phi(z(t ; z(0)))| & =\left|\mathbb{E}\left[\mathbb{E}\left(\phi(z(t+s ; z(0))) \mid \mathcal{F}_{s}\right)\right]-\mathbb{E} \phi(z(t ; z(0)))\right| \\
& =\left|\int_{R_{+}^{2}} \mathbb{E} \phi(z(t ; \tilde{z}(0))) p(s, z(0), \mathrm{d} \tilde{z}(0))-\mathbb{E} \phi(z(t ; z(0)))\right| \\
& \leqslant \int_{R_{+}^{2}}|\mathbb{E} \phi(z(t ; \tilde{z}(0)))-\mathbb{E} \phi(z(t ; z(0)))| p(s, z(0), \mathrm{d} \tilde{z}(0)) .
\end{aligned}
$$


In view of (4.1), there exists a $t_{1}>0$ such that for $t \geqslant t_{1}$,

$$
|\mathbb{E} \phi(z(\mathrm{t} ; \tilde{z}(0)))-\mathbb{E} \phi(z(\mathrm{t} ; z(0)))| \leqslant \mathbb{E}|\phi(z(\mathrm{t} ; \tilde{z}(0)))-\phi(z(\mathrm{t} ; z(0)))| \leqslant \mathbb{E}|z(\mathrm{t} ; \tilde{z}(0))-z(\mathrm{t} ; z(0))| \leqslant \varepsilon .
$$

Plugging (4.3) into (4.2) leads to

$$
|\mathbb{E} \phi(z(t+s ; z(0)))-\mathbb{E} \phi(z(t ; z(0)))| \leqslant \varepsilon, \forall t \geqslant t_{1}, s>0 .
$$

It then follows from the arbitrariness of $\phi$ that

$$
\sup _{\phi \in \Phi}|\mathbb{E} \phi(z(t+s ; z(0)))-\mathbb{E} \phi(z(t ; z(0)))| \leqslant \varepsilon, \forall t \geqslant t_{1}, s>0 .
$$

That is to say

$$
\mathrm{d}_{\Phi}(\mathrm{p}(\mathrm{t}+\mathrm{s}, z(0), \cdot), \mathrm{p}(\mathrm{t}, z(0), \cdot)) \leqslant \varepsilon \text { for all } t \geqslant \mathrm{t}_{1}, \mathrm{~s}>0 .
$$

Consequently, for all $z(0) \in R_{+}^{2},\{p(t, z(0), \cdot)\}$ is Cauchy with respect to $t$. Choose $z(0)=(0.5,0.5)^{\mathrm{T}}$, then $\left\{\mathrm{p}\left(\mathrm{t},(0.5,0.5)^{\mathrm{T}}, \cdot\right)\right\}$ is Cauchy. That is to say, there is a unique $\chi(\cdot) \in \mathscr{P}\left(\mathrm{R}_{+}^{2}\right)$ satisfying

$$
\lim _{t \rightarrow+\infty} d_{\Phi}\left(p\left(t,(0.5,0.5)^{\mathrm{T}}, \cdot\right), \chi(\cdot)\right)=0 .
$$

Thanks to (4.1),

$$
\lim _{t \rightarrow+\infty} d_{\Phi}\left(p(t, z(0), \cdot), p\left(t,(0.5,0.5)^{\mathrm{T}}, \cdot\right)\right)=0
$$

Therefore,

$$
\begin{aligned}
\lim _{\mathrm{t} \rightarrow+\infty} \mathrm{d}_{\Phi}(\mathrm{p}(\mathrm{t}, z(0), \cdot), \chi(\cdot)) \leqslant & \lim _{\mathrm{t} \rightarrow+\infty} \mathrm{d}_{\Phi}\left(\mathrm{p}(\mathrm{t}, z(0), \cdot), \mathrm{p}\left(\mathrm{t},(0.5,0.5)^{\mathrm{T}}, \cdot\right)\right) \\
& +\lim _{\mathrm{t} \rightarrow+\infty} \mathrm{d}_{\Phi}\left(\mathrm{p}\left(\mathrm{t},(0.5,0.5)^{\mathrm{T}}, \cdot\right), \chi(\cdot)\right)=0 .
\end{aligned}
$$

On the other hand, since Assumption 3.1 holds, then $M>0$. It then follows from (3.3) that the support of $\chi$ is $R_{+}^{2}$. This completes the proof.

Remark 4.4. Under Assumptions 3.1 and 4.2, according to Theorem 3.4 we can see that if $M_{2}<0$, then model (2.2) is not SID.

\section{Optimal harvesting policy}

Now let us consider the optimal harvesting policy of model (2.2). Our objective is to find out the optimal harvesting effect $h^{*}=\left(h_{1}^{*}, h_{2}^{*}\right)^{T}$ such that:

- $\mathrm{Y}(\mathrm{h})=\lim _{\mathrm{t} \rightarrow+\infty} \sum_{i=1}^{2} \mathbb{E}\left(h_{\mathrm{i}} z_{\mathrm{i}}(\mathrm{t})\right)$ is maximum;

Both $z_{1}$ and $z_{2}$ are persistent.

Theorem 5.1. Consider system (2.2), and let Assumptions 3.1 and 4.2 hold. Define

$$
\bar{M}=\left(\begin{array}{cc}
m_{11} & -m_{12} \\
-m_{21} & m_{22}
\end{array}\right), L=\left(r_{1}-\eta_{1},-r_{2}-\eta_{2}\right)^{\mathrm{T}}, \Delta=\left(\Delta_{1}, \Delta_{2}\right)^{\mathrm{T}}=\left[\bar{M}\left(\bar{M}^{-1}\right)^{\mathrm{T}}+\mathrm{I}\right]^{-1} \mathrm{~L} .
$$

(i') If $\left.M_{2}\right|_{h=\Delta}>0, \Delta_{1} \geqslant 0, \Delta_{2} \geqslant 0$ and $\bar{M}^{-1}+\left(\bar{M}^{-1}\right)^{\mathrm{T}}$ is positive semi-definite, then $\mathrm{h}^{*}=\Delta$ and

$$
\mathrm{Y}^{*}=\max \{\mathrm{Y}(\mathrm{h})\}=\Delta^{\mathrm{T}} \bar{M}^{-1}(\mathrm{~L}-\Delta) .
$$


(ii') If the conditions in (i') are not satisfied, then the optimal harvesting policy does not exist.

Proof. Let $H=\left\{h=\left(h_{1}, h_{2}\right)^{T} \in R^{2} \mid M_{2}>0, h_{i} \geqslant 0, i=1,2\right\}$. According to Lemma 3.2 and Remark 3.5, if $h \in H$, then (3.3) is satisfied; moreover, if $h^{*}$ exists, then $h^{*} \in H$.

(i'). By (3.3), for all $h \in H$,

$$
\lim _{\mathrm{t} \rightarrow+\infty} \mathrm{t}^{-1} \int_{0}^{\mathrm{t}} \mathrm{h}^{\mathrm{T}} z(\mathrm{~s}) \mathrm{d} s=\sum_{\mathrm{i}=1}^{2} \mathrm{~h}_{\mathrm{i}} \lim _{\mathrm{t} \rightarrow+\infty} \mathrm{t}^{-1} \int_{0}^{\mathrm{t}} z_{\mathrm{i}}(\mathrm{s}) \mathrm{d} s=\mathrm{h}^{\mathrm{T}} \bar{M}^{-1}(\mathrm{~L}-\mathrm{h}) .
$$

By Theorem 4.3, there is a unique invariant measure $\chi(\cdot)$ to model (2.2). According to Corollary 3.4.3 and Theorem 3.2.6 in [11], $\chi(\cdot)$ is strong mixing and ergodic. By (3.3.2) in [11], one obtains

$$
\lim _{t \rightarrow+\infty} t^{-1} \int_{0}^{t} h^{T} z(s) d s=\int_{R_{+}^{2}} h^{T} y x(d y) .
$$

Denote by $\zeta(y)$ the stationary probability density of model (2.2). Then

$$
Y(h)=\lim _{t \rightarrow+\infty} \sum_{i=1}^{2} \mathbb{E}\left(h_{i} z_{i}(t)\right)=\lim _{t \rightarrow+\infty} \mathbb{E}\left(h^{T} z(t)\right)=\int_{R_{+}^{2}} h^{T} y \zeta(y) d y .
$$

Since the invariant measure of model (2.2) is unique, then according to the one-to-one correspondence between $\zeta(\cdot)$ and $\chi(\cdot)$ (see e.g., [11, P.105]), we have

$$
\int_{R_{+}^{2}} h^{\mathrm{T}} y \zeta(y) d y=\int_{R_{+}^{2}} h^{\mathrm{T}} y x(d y) .
$$

In view of (5.2), (5.3), (5.4), and (5.5), one can see that

$$
Y(h)=h^{T} \bar{M}^{-1}(L-h) .
$$

Denote by $\Delta=\left(\Delta_{1}, \Delta_{2}\right)^{\top}$ the solution of the following equation

$$
0=\frac{d Y(h)}{d h}=\frac{d\left(h^{T}\right)}{d h} \bar{M}^{-1}(L-h)+\frac{d}{d h}\left[(L-h)^{T}\left(\bar{M}^{-1}\right)^{T}\right] h=\bar{M}^{-1} L-\left[\bar{M}^{-1}+\left(\bar{M}^{-1}\right)^{T}\right] h .
$$

Then $\Delta=\left[\bar{M}\left(\bar{M}^{-1}\right)^{\mathrm{T}}+\mathrm{I}\right]^{-1} \mathrm{~L}$. It is easy to see that

$$
\frac{\mathrm{d}}{\mathrm{dh}}\left[\frac{\mathrm{d} Y(\mathrm{~h})}{\mathrm{dh}}\right]=\left(\frac{\mathrm{d}}{\mathrm{dh}}\left[\left(\frac{\mathrm{d} Y(\mathrm{~h})}{\mathrm{dh}}\right)^{\mathrm{T}}\right]\right)^{\mathrm{T}}=\left(\frac{\mathrm{d}}{\mathrm{dh}}\left[\mathrm{L}^{\mathrm{T}}\left(\bar{M}^{-1}\right)^{\mathrm{T}}-\mathrm{h}^{\mathrm{T}}\left[\bar{M}^{-1}+\left(\bar{M}^{-1}\right)^{\mathrm{T}}\right]\right]\right)^{\mathrm{T}}=-\left(\bar{M}^{-1}+\left(\bar{M}^{-1}\right)^{\mathrm{T}}\right) .
$$

Therefore, if $\bar{M}^{-1}+\left(\bar{M}^{-1}\right)^{\mathrm{T}}$ is positive semi-definite and $\Delta \in \mathrm{H}$, then $\Delta$ is the unique extreme point of $\mathrm{Y}(\mathrm{h})$, and hence $\mathrm{h}^{*}=\Delta$ and (5.1) holds.

(ii'). Firstly, let us demonstrate that if $\left.M_{2}\right|_{h=\Delta} \leqslant 0$, or $\Delta_{1}<0$, or $\Delta_{2}<0$, then there is no optimal harvesting policy. If this statement is false, denote by $\widetilde{\mathrm{h}}^{*}=\left(\widetilde{\mathrm{h}}_{1}^{*}, \widetilde{\mathrm{h}}_{2}^{*}\right)^{\mathrm{T}}$ the optimal harvesting effort. Hence $\widetilde{\mathrm{h}}^{*} \in \mathrm{H}$. Therefore,

$$
\left.M_{2}\right|_{h=\widetilde{h}^{*}}>0, \quad \widetilde{h}_{1}^{*} \geqslant 0, \quad \widetilde{h}_{2}^{*} \geqslant 0 .
$$

Note that $\widetilde{\mathrm{H}}^{*}$ is the optimal harvesting effort, hence $\widetilde{\mathrm{H}}^{*}$ must be the unique solution of (5.7). On the other hand, $\Delta$ is also the solution of (5.7). Consequently, $\widetilde{h}_{i}^{*}=\Delta_{i} \geqslant 0$ and

$$
\left.\mathrm{M}_{2}\right|_{\mathrm{h}=\Delta}=\left.\mathrm{M}_{2}\right|_{\mathrm{h}=\widetilde{\mathrm{h}}^{*}}>0 .
$$

This is a contradiction.

Secondly, let us demonstrate that if $\left.M_{2}\right|_{h=\Delta}>0, \Delta_{1} \geqslant 0, \Delta_{2} \geqslant 0$, and $\bar{M}^{-1}+\left(\bar{M}^{-1}\right)^{\mathrm{T}}$ is not positive semi-definite, then there is no optimal harvesting policy. Note that $\left.M_{2}\right|_{h=\Delta}>0, \Delta_{1} \geqslant 0, \Delta_{2} \geqslant 0$, then we 
have (5.6). Let $\left(\kappa_{i j}\right)_{2 \times 2}=\bar{M}^{-1}+\left(\bar{M}^{-1}\right)^{\mathrm{T}}$. Hence

$$
\kappa_{11}=2 m_{22} / M>0 .
$$

It follows that $\bar{M}^{-1}+\left(\bar{M}^{-1}\right)^{\mathrm{T}}$ is not a negative semi-definite matrix. Then $\bar{M}^{-1}+\left(\bar{M}^{-1}\right)^{\mathrm{T}}$ is indefinite. Consequently, $\mathrm{Y}(\mathrm{h})$ in (5.6) does not have extreme points ([4]).

\section{Numerical simulations and conclusions}

In this paper, we proposed and studied a stochastic service-resource mutualism system with harvesting and Lévy jumps. Our main results are Theorems 3.4, 4.3, and 5.1. Theorem 3.4 gives the sufficient and necessary conditions for persistence in the mean and extinction of the species. Theorem 4.3 studies the SID of the model. Theorem 5.1 establishes the sufficient and necessary conditions for the existence of the optimal harvesting policy, and gives the explicit forms of the optimal harvesting effect and the maximal of the yield.

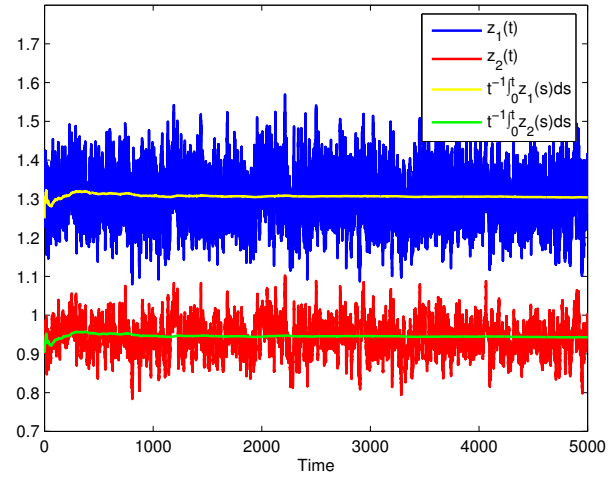

(a)

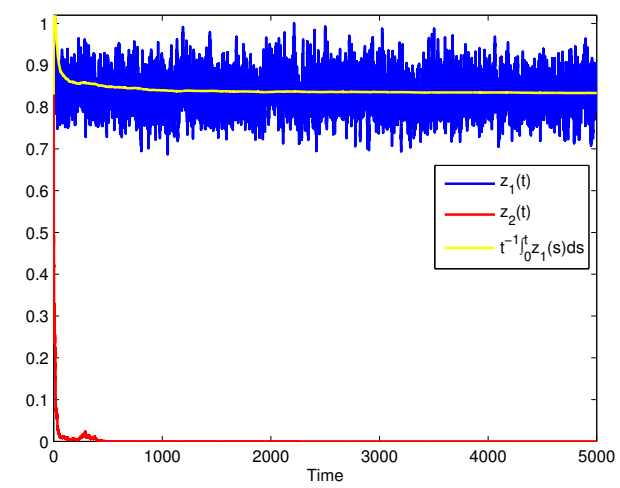

(b)

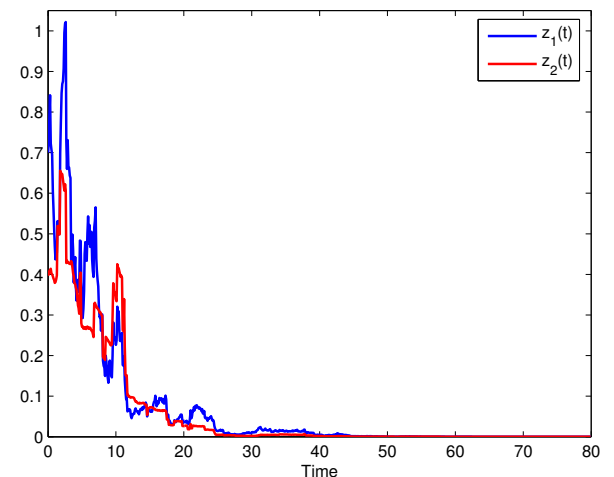

(c)

Figure 1: Solutions of (2.2) with $r_{1}=0.6, r_{2}=0.03, h_{1}=h_{2}=0, m_{11}=0.6, m_{12}=0.3, m_{21}=0.4, m_{22}=0.5, \mathbb{X}=R_{+}, \beta(\mathbb{X})=1$, $\xi_{1}(x) \equiv 0.3504, \xi_{2}(x) \equiv 0.1482$. (a) is with $\sigma_{1}^{2}=0.1, \sigma_{2}^{2}=0.02$; (b) is with $\sigma_{1}^{2}=0.1, \sigma_{2}^{2}=0.62$; (c) is with $\sigma_{1}^{2}=1.2, \sigma_{2}^{2}=0.02$.

Theorem 3.4 reveals that whether $z_{1}$ (respectively, $z_{2}$ ) is persistent or not depends only upon the sign of $b_{1}$ (respectively, $M_{2}$ ) which has close relationship with the white noises and Lévy noises.

- Firstly, let us consider the effects of the white noises. Clearly,

$$
\frac{\mathrm{db}_{1}}{\mathrm{~d}\left(\sigma_{1}^{2}\right)}<0, \frac{\mathrm{db}_{1}}{\mathrm{~d}\left(\sigma_{2}^{2}\right)}=0, \frac{\mathrm{dM}}{\mathrm{d}\left(\sigma_{1}^{2}\right)}<0, \frac{\mathrm{d} M_{2}}{\mathrm{~d}\left(\sigma_{2}^{2}\right)}<0 .
$$

Hence, with the increase of intensity of white noise of the resource species, $\sigma_{1}^{2}$, both the resource species and the service species tend to die out; with the increase of intensity of white noise of the 
service species, $\sigma_{2}^{2}$, the service species tends to die out while the persistence of the resource species does not change. To see these more clearly, let us work our several numerical simulations. In Fig. 1, we only let the values of $\sigma_{1}^{2}$ and $\sigma_{2}^{2}$ vary. Comparing Fig. 1 (a) with Fig. 1 (c), we can see that with the increase of $\sigma_{1}^{2}$ (from 0.1 to 1.2), both $z_{1}$ and $z_{2}$ tend to die out. On the other hand, comparing Fig. 1 (a) with Fig. 1 (b), one can observe that with the increase of $\sigma_{2}^{2}$ (from 0.02 to 0.62 ), $z_{2}$ tends to die out while $z_{1}$ still is persistent.

- Now let us see the effects of the Lévy noises. For simplicity, let $\xi_{i}(x) \equiv \tilde{\xi}_{i}>-1, i=1,2$. Note that

$$
\frac{\mathrm{db}}{\mathrm{d} \tilde{\xi}_{1}}\left\{\begin{array}{ll}
<0, & \text { if } \tilde{\xi}_{1}>0, \\
>0, & \text { if } \tilde{\xi}_{1}<0,
\end{array} \quad \frac{\mathrm{db}}{\mathrm{d} \tilde{\xi}_{2}}=0, \frac{\mathrm{d} M_{2}}{\mathrm{~d} \tilde{\xi}_{1}}\left\{\begin{array} { l l } 
{ < 0 , } & { \text { if } \tilde { \xi } _ { 1 } > 0 , } \\
{ > 0 , } & { \text { if } \tilde { \xi } _ { 1 } < 0 , }
\end{array} \quad \frac { \mathrm { d } M _ { 2 } } { \mathrm { d } \tilde { \xi } _ { 2 } } \left\{\begin{array}{ll}
<0, & \text { if } \tilde{\xi}_{2}>0, \\
>0, & \text { if } \tilde{\xi}_{2}<0 .
\end{array}\right.\right.\right.
$$

Here we only consider the case $\tilde{\xi}_{i}>0, i=1,2$, other cases can be analyzed similarly. In this case, (6.2) means that with the increase of intensity of Lévy noise of the resource species, both the resource species and the service species tend to die out; with the increase of intensity of Lévy noise of the service species, the service species tends to die out while the persistence of the resource species does not change.

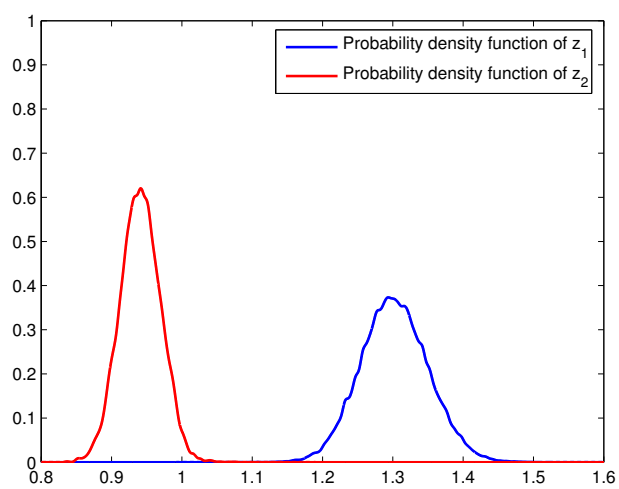

Figure 2: Probability density function of (2.2) at $t=5000$. The parameters are the same with these in Fig. 1 (a).

Theorem 4.3 and Remark 4.4 indicate that whether model (2.2) is SID or not depends only upon the sign of $M_{2}$. According to (6.1), we can see that both $\sigma_{1}^{2}$ and $\sigma_{2}^{2}$ are harmful for the SID of model (2.2). Similarly, in view of (6.2), when $\tilde{\xi_{i}}>0, i=1,2$, both $\tilde{\xi_{1}}$ and $\tilde{\xi_{2}}$ are also harmful for the SID of model (2.2). We plot the probability density functions of $\left(z_{1}(t), z_{2}(t)\right)^{T}$ at time $t=5000$ in Fig. 2 where the parameters are the same with these in Fig. 1 (a).

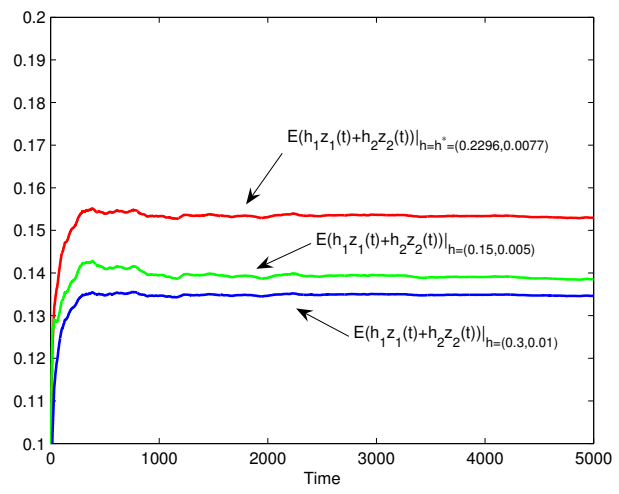

Figure 3: $\mathbb{E}\left[h_{1} z_{1}(t)+h_{2} z_{2}(t)\right]$ of (2.2) with the same parameters given in Fig. 1 (a) except $h$. Red line is with $h=h^{*}=$ $(0.2296,0.0077)^{\mathrm{T}}$, green line is with $\mathrm{h}=(0.15,0.005)^{\mathrm{T}}$, and blue line is with $\mathrm{h}=(0.3,0.01)^{\mathrm{T}}$. 
Theorem 5.1 gives the optimal harvesting policy of model (2.2). Let us introduce an example to illustrate this. Consider the parameters given in Fig. 1 (a) except $h$. It is easy to see that Assumptions 3.1 and 4.2 hold, and $\bar{M}^{-1}+\left(\bar{M}^{-1}\right)^{\mathrm{T}}$ is positive definite. According to Theorem 5.1, compute that

$$
\Delta=(0.2296,0.0077)^{\mathrm{T}},\left.\mathrm{M}_{2}\right|_{\mathrm{h}=\Delta}=0.0735>0 .
$$

That is to say, the conditions in (i') are satisfied. Hence

$$
\mathrm{h}^{*}=\Delta=(0.2296,0.0077)^{\mathrm{T}}, \mathrm{Y}^{*}=0.1535 \text {. }
$$

We plot $\mathbb{E}\left[h_{1} z_{1}(t)+h_{2} z_{2}(t)\right]$ in Fig. 3. Red line is with $h=h^{*}=(0.2296,0.0077)^{\mathrm{T}}$, green line is with $h=(0.15,0.005)^{\mathrm{T}}$, and blue line is with $\mathrm{h}=(0.3,0.01)^{\mathrm{T}}$. Clearly, when $\mathrm{h}=\mathrm{h}^{*}$, the yield is maximum. On the other hand, Theorem 5.1 shows that both the optimal harvesting effect $h^{*}=\left(h_{1}^{*}, h_{2}^{*}\right)^{\mathrm{T}}$ and the maximal of yield $Y^{*}$ have close relationships with $\sigma_{i}^{2}$ and $\xi_{i}(x), i=1,2$. Once again, we let $\xi_{i}(x) \equiv \tilde{\xi}_{i}>0, i=1,2$. Compute that

$$
\frac{d h_{i}^{*}}{\mathrm{~d}\left(\sigma_{j}^{2}\right)}<0, \frac{d h_{i}^{*}}{\mathrm{~d} \tilde{\xi}_{j}}<0, \frac{\mathrm{d} Y^{*}}{\mathrm{~d}\left(\sigma_{j}^{2}\right)}<0, \frac{\mathrm{d} Y^{*}}{\mathrm{~d} \tilde{\xi}_{j}}<0, i, j=1,2
$$

Therefore, with the increase of $\sigma_{i}^{2}$ and $\tilde{\xi_{i}}$, both $h_{j}^{*}$ and $Y^{*}$ decrease, $i, j=1,2$.

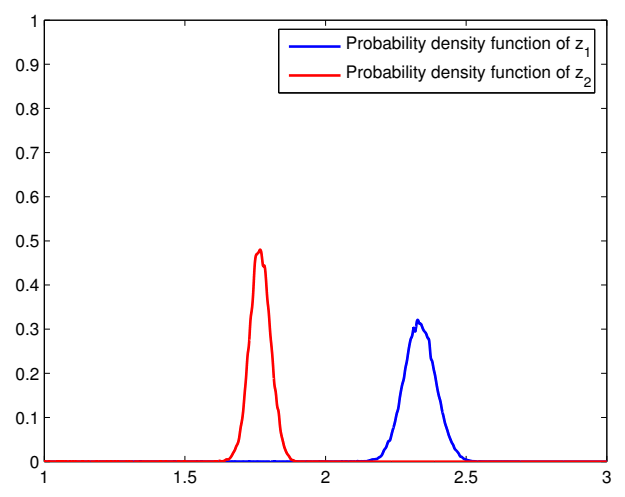

Figure 4: Probability density function of (2.2) at $t=5000$. The parameters are the same with these in Fig. 1 (a) except $m_{12}=0.51$.

Some problems deserve further research. Firstly, it is worth weakening Assumption 3.1 and Assumption 4.2. It is important to mention that Assumption 3.1 and Assumption 4.2 are just sufficient. For example, Fig. 4 indicates that even $m_{22}<m_{12}$, system (2.2) could be SID. It is also interesting to take other factors into account, for example, regime switching (see e.g., [28]), and reaction-diffusion (see e.g., [1]). We leave these problems for future work.

\section{Acknowledgment}

The authors thank the editor and the anonymous referee for their valuable comments. This work was supported by the National Natural Science Foundation of P. R. China [Nos. 11771174 and 11301207]; Project Fund from China Postdoctoral Science Foundation (Nos. 2015M571349 and 2016T90236); Natural Science Foundation of Jiangsu Province [No. BK20170067]; Jiangsu Province "333 High-level Personnel Training Project" and "333 Project" Research Foundation in Jiangsu Province [No. BRA2017476]; Jiangsu College Students Innovative Entrepreneurial Training Program [No. 201710323007Z].

\section{References}

[1] C.-Z. Bai, Multiplicity of solutions for a class of non-local elliptic operators systems, Bull. Korean Math. Soc., 54 (2017), 715-729. 6

[2] J.-H. Bao, C.-G. Yuan, Stochastic population dynamics driven by Lévy noise, J. Math. Anal. Appl., 391 (2012), $363-375$. 2,3 
[3] I. Barbălat, Systémes d'équations différentielles d'oscillations non linéaires, (French) Rev. Math. Pures Appl., 4 (1959), 267-270. 4

[4] M. S. Bazaraa, C. M. Shetty, Nonlinear programming, Theory and algorithms, John Wiley \& Sons, New YorkChichester-Brisbane, (1979) 5

[5] J. R. Beddington, R. M. May, Harvesting natural populations in a randomly fluctuating environment, Science, 197 (1977), 463-465. 1, 2

[6] M. Begon, J. L. Harper, C. R. Townsend, Ecology, Individuals, populations and communities, Blackwell scientific publications, Cambridge, (1986). 1

[7] C. A. Braumann, Variable effort harvesting models in random environments: generalization to density-dependent noise intensities, Deterministic and stochastic modeling of biointeraction, West Lafayette, IN, (2000), Math. Biosci., 177/178 (2002), 229-245. 2

[8] E. Braverman, L. Braverman, Optimal harvesting of diffusive models in a nonhomogeneous environment, Nonlinear Anal., 71 (2009), e2173-e2181. 1

[9] E. Braverman, R. Mamdani, Continuous versus pulse harvesting for population models in constant and variable environment, J. Math. Biol., 57 (2008), 413-434. 1

[10] C. W. Clark, Mathematical bioeconomics, The optimal management of renewable resources, Second edition, With a contribution by Gordon Munro, Pure and Applied Mathematics (New York), A Wiley-Interscience Publication, John Wiley \& Sons, Inc., New York, (1990). 1, 2

[11] G. Da Prato, J. Zabczyk, Ergodicity for infinite-dimensional systems, London Mathematical Society Lecture Note Series, Cambridge University Press, Cambridge, (1996). 5, 5

[12] M. Fan, K. Wang, Optimal harvesting policy for single population with periodic coefficients, Math. Biosci., 152 (1998), 165-177. 1

[13] T. C. Gard, Stability for multispecies population models in random environments, Nonlinear Anal., 10 (1986), 1411-1419. 2

[14] T. K. Kar, Modelling and analysis of a harvested prey-predator system incorporating a prey refuge, J. Comput. Appl. Math., 185 (2006), 19-33. 1

[15] H. Kunita, Itô's stochastic calculus: its surprising power for applications, Stochastic Process. Appl., 120 (2010), $622-652$. 3,4

[16] M. Liu, C.-Z. Bai, Optimal harvesting of a stochastic logistic model with time delay, J. Nonlinear Sci., 25 (2015), 277-289. 1

[17] M. Liu, C.-Z. Bai, Analysis of a stochastic tri-trophic food-chain model with harvesting, J. Math. Biol., 73 (2016), 597-625. 2

[18] M. Liu, C.-Z. Bai, Dynamics of a stochastic one-prey two-predator model with Léry jumps, Appl. Math. Comput., 284 (2016), 308-321. 2

[19] M. Liu, C.-Z. Bai, Optimal harvesting of a stochastic delay competitive model, Discrete Contin. Dyn. Syst. Ser. B, 22 (2017), 1493-1508. 1

[20] M. Liu, X. He, J. Yu, Dynamics of a stochastic regime-switching predator-prey model with harvesting and distributed delays, Nonlinear Anal. Hybrid Syst., 28 (2018), 87-104. 1

[21] L.-D. Liu, X.-Z. Meng, T.-H. Zhang, Optimal control strategy for an impulsive stochastic competition system with time delays and jumps, Phys. A, 477 (2017), 99-113. 1, 2

[22] M. Liu, K. Wang, Stochastic Lotka-Volterra systems with Lévy noise, J. Math. Anal. Appl., 410 (2014), 750-763. 3, 3.3

[23] X.-R. Mao, C.-G. Yuan, Stochastic differential equations with Markovian switching, Imperial College Press, London, (2006). 4

[24] W. M. Murdoch, C. J. Briggs, R. M. Nisbet, Consumer-resource dynamics, Princeton University Press, Princeton, (2003). 2

[25] Y.-S. Wang, D. L. DeAngelis, J. N. Holland, Uni-directional consumer-resource theory characterizing transitions of interaction outcomes, Ecol. Complex., 8 (2011), 249-257. 2

[26] Y. Zhao, S.-L. Yuan, Optimal harvesting policy of a stochastic two-species competitive model with Lévy noise in a polluted environment, Phys. A, 477 (2017), 20-33. 1, 2

[27] Y. Zhu, M. Liu, Permanence and extinction in a stochastic service-resource mutualism model, Appl. Math. Lett., 69 (2017), 1-7. 2

[28] C. Zhu, G. Yin, On hybrid competitive Lotka-Volterra ecosystems, Nonlinear Anal., 71 (2009), e1370-e1379. 6

[29] X.-L. Zou, W.-X. Li, K. Wang, Ergodic method on optimal harvesting for a stochastic Gompertz-type diffusion process, Appl. Math. Lett., 26 (2013), 170-174. 1

[30] X.-L. Zou, K. Wang, Optimal harvesting for a logistic population dynamics driven by a Lévy process, J. Optim. Theory. Appl., 161 (2014), 969-979. 1 